\title{
Safety Study of Transcranial Static Magnetic Field Stimulation (tSMS) of the Human Cortex
}

\author{
A. Oliviero , M.C. Carrasco-López , M. Campolo , Y.A. Perez-Borrego , V. Soto-León , \\ J.J. Gonzalez-Rosa , A.M. Higuero , B.A. Strange , J. Abad-Rodriguez , G. Foffani
}

\begin{abstract}
A B S T R A C T
Background: Transcranial static magnetic field stimulation (tSMS) in humans reduces cortical excitability. Objective: The objective of this study was to determine if prolonged tSMS ( $2 \mathrm{~h}$ ) could be delivered safely in humans. Safety limits for this technique have not been described.

Methods: tSMS was applied for $2 \mathrm{~h}$ with a cylindric magnet on the occiput of 17 healthy subjects. We assessed tSMS-related safety aspects at tissue level by measuring levels of neuron-specific enolase (NSE, a marker of neuronal damage) and S100 (a marker of glial reactivity and damage). We also included an evaluation of cognitive side effects by using a battery of visuomotor and cognitive tests.

Results: tSMS did not induce any significant increase in NSE or S100. No cognitive alteration was detected. Conclusions: Our data indicate that the application of tSIMS is safe in healthy human subjects, at least within these parameters.
\end{abstract}

\section{Introduction}

Non-invasive brain stimulation (NIBS) techniques have made an important contribution to cognitive neuroscience and have been proposed as a treatment for neuropsychiatric disorders [1]. Repetitive transcranial magnetic stimulation (rTMS) and transcranial direct current stimulation (tDCS) are commonly used for NIBS in humans and animals. Recently we described that the application of transcranial static magnetic field stimulation (tSMS) in humans reduces the output of motor cortex - tested using TMS - for a few minutes after the end of stimulation [2]. Reduced motor output after tSMS can be explained by reduced motor cortex excitability. These results have been recently replicated by a different group [3]. tSMS using small magnets may thus be a promising tool to modulate cerebral excitability in a non-invasive, painless and reversible way.

Static magnetic fields, unlike time-varying magnetic fields, are not associated with induced electric currents and have been shown to influence a variety of biological systems [4]. A number of studies suggest that static magnetic fields act primarily at the synapse and alter the function of membrane ion channels [5], and the application of static magnetic fields to different animal preparations seem to have an effect that outlasts the time of stimulation [6]. When tSMS is applied in humans, the cortex is at least $2 \mathrm{~cm}$ away, so most of the strength of the magnetic field will not reach the target. The recommended limits by the World Health Organization (WHO) about safe exposure to static magnet fields are "time weighted average of $200 \mathrm{mT}$ during the working day for occupational 


\section{Experimental setup}

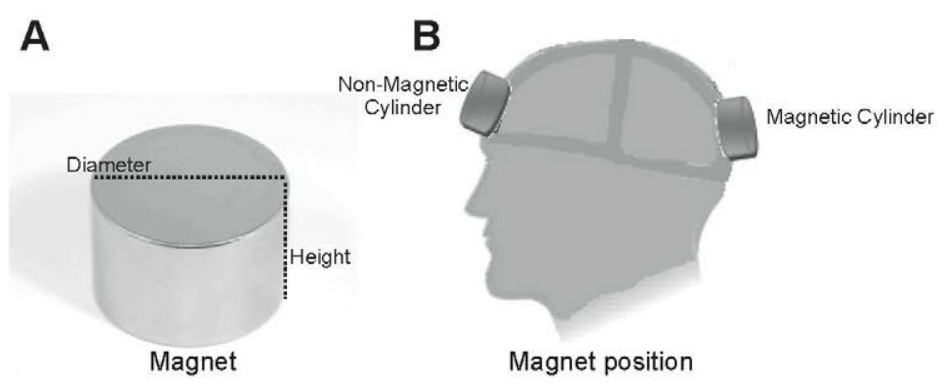

C

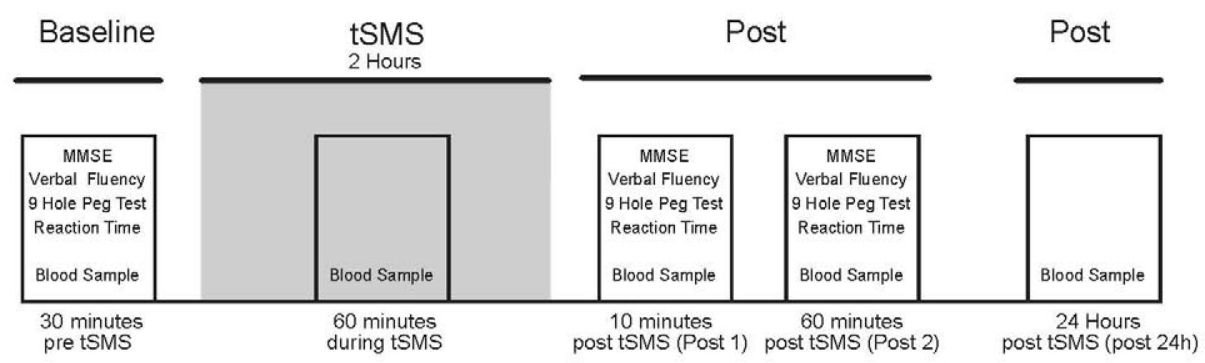

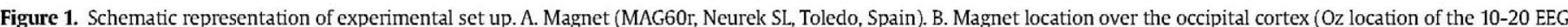

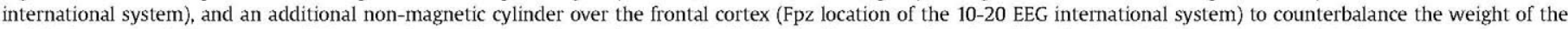
occipital magnet. C. Time course of the experiment. The experimental protocol is divided in five time points: baseline, during tSMS, post1, post 2 and 24 h.

exposure" (i.e. 8 h/day, 5 days/week) (http://www.who.int/pehemf/publications/facts/fs299/en/index.html). With the magnets we normally use in our lab (MAG45r and MAG60r; Neurek SL, Toledo, Spain), at $2 \mathrm{~cm}$ from the axis (the approximate distance from the scull to the cortex) the magnetic field is around 150-200 mT [7], so within the safe limit proposed by the WHO. Moreover, many studies about safety of magnetic resonance imaging (MRI) techniques consider safe an exposure to magnetic fields $>8 \mathrm{~T}$ for experimental sessions that can last hours [8]. On the other hand, it cannot be excluded that the different gradient shape and/or the different magnetic field orientation may have different safety profiles.

The purpose of this study was to test the safety of prolonged tSMS $(2 \mathrm{~h})$ of the occipital cortex in healthy volunteers to establish safety guidelines for future tSMS experiments and therapeutic trials. We tested the effects of tSMS on a cellular level, by measuring serum levels of neuron-specific enolase (NSE) and protein S-100 [9] - sensitive markers for neural or glial brain damage - in healthy volunteers before, during and after tSMS. In order to provide further evidence for the safety of tSMS, a battery of neuropsychological tests were performed to exclude cognitive adverse effects. Specifically, we chose the Mini Mental State Exam (MMSE) as an evaluation of global cognitive state [10], the Nine-Hole peg test (NHPT) to evaluate a fine motor task and visuomotor coordination, a two-choice reaction time test to assay attentional levels (and again visuomotor coordination). Verbal fluency, a cognitive process considered to be primarily frontal lobe-dependent, was tested to determine cognitive function associated to a brain location distant from the stimulated area [11].

\section{Methods}

\section{Subjects}

Seventeen healthy volunteers participated in this study (10 males; mean age $34.4+7.3$ years; age range $24-45$ years).
Exclusion criteria were significant medical or psychiatric illness, pregnancy and concurrent use of neuroactive drugs. We also excluded individuals with pacemakers, brain stimulators, medication pumps or any type of metal object in the head including eyes except for dental appliances or fillings - which might pose a physical hazard during tSMS. All subjects but one were right handed according to the Edinburgh handedness inventory [12]. The study was approved by the local ethical committee. Informed consent was obtained from all subjects.

\section{Experimental set-up}

Experimental set-up is shown schematically in Fig. 1.

\section{tSMS procedures}

To deliver tSMS we used a cylindrical Nickel-plated (Ni-Cu-Ni) $\mathrm{NdFeB}$ magnet of $60 \mathrm{~mm}$ diameter, $30 \mathrm{~mm}$ of thickness and a weight of $370 \mathrm{~g}$ (MAG60r, Neurek SL, Toledo, Spain). North magnetic field polarity was used (i.e. north pole was placed over the scalp). During the experiment, all subjects had tSMS over the occipital cortex $(\mathrm{Oz}$ location of the 10-20 EEG international system), and an additional non-magnetic cylinder was located over the frontal cortex (Fpz location of the 10-20 EEG international system) and remained fixed during the whole experiment to counterbalance the weight of the occipital cylinder. The non-magnetic cylinder was a steel nickelcoated cylinder, had the same size, a weight of $368 \mathrm{~g}$ similar to the MAG60r (MAG60s, Neurek SL, Toledo, Spain). The cylinders were held in place with a leather strapping system (MAGlet60+, Neurek $\mathrm{SL}$, Toledo, Spain). The tSMS was applied for $2 \mathrm{~h}$.

\section{Blood samples for determining NSE and S-100}

We measured serum concentrations of NSE and S100, as sensitive markers of neuronal damage and glial activation, respectively. Blood samples were taken in EDTA-free tubes from each subject at 

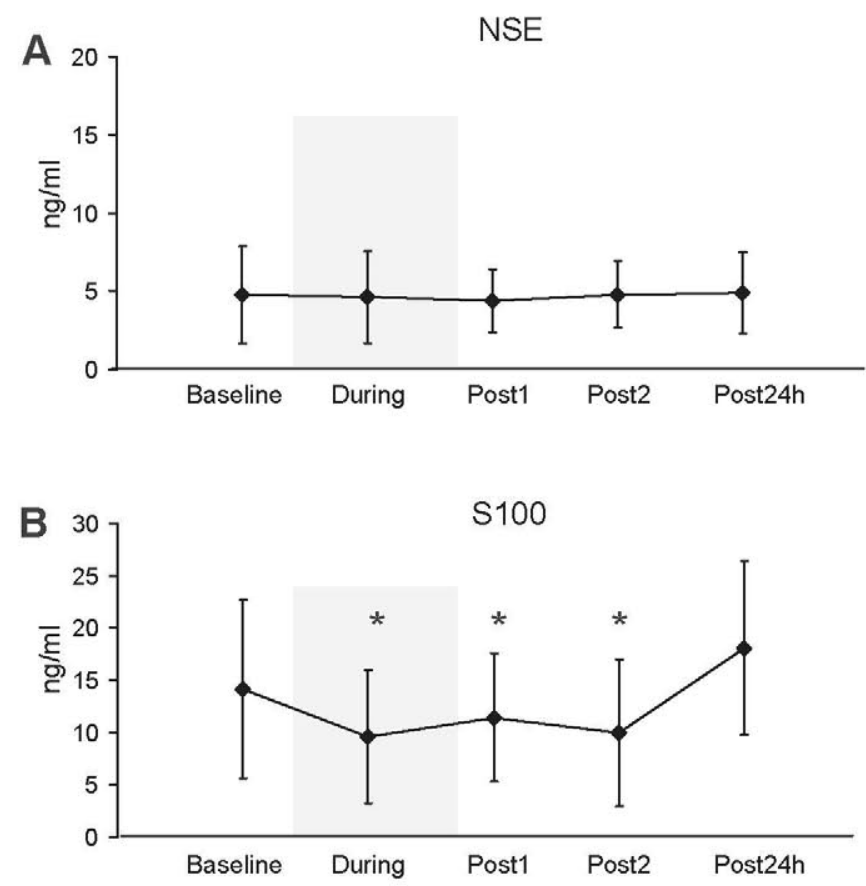

Figure 2. Time course and concentration of serum levels of neuron-specific enolase (NSE) and protein S-100. A. Time course of NSE concentration $(\mathrm{ng} / \mathrm{ml})$. B. Time course of $S-100$ concentration $(\mathrm{ng} / \mathrm{ml}) .^{*}=P<0.05$ (paired $t$-test). Error bars indicate standard deviations.

baseline before the tSMS treatment was started (baseline), after 60 min during the treatment (during), immediately after (post 1 ), $60 \mathrm{~min}$ after (post2), and $24 \mathrm{~h}$ (only 13 subjects) after the end of tSMS (post $24 \mathrm{~h}$ ). A peripheral venous catheter in the left cubital fossa (or dorsum of the forearm) was maintained throughout the experiment to allow serial blood drawing (with the exception of the $24 \mathrm{~h}$ blood sample that was obtained via new venipuncture). Samples were left $1 \mathrm{~h}$ at room temperature and 3 more hours at $4{ }^{\circ} \mathrm{C}$ to induce clot retraction. Crude sera were obtained by low-speed centrifugation $(800 \times g)$, and cleared-up by medium-speed centrifugation $(5000 \times \mathrm{g})$. Aliquots were preserved at $-20^{\circ} \mathrm{C}$ until used. Serum NSE measurements were performed using the Quantikine ${ }^{(i)}$ ELISA kit for Human Enolase 2/Neuron-specific Enolase (R\&D Systems). S100 quantification was performed using the Human S100B ELISA kit (Millipore). In both cases, manufacturers' instructions were followed.

In one subject, it was not possible to obtain blood samples during tSMS and in post 1 . In another subject, it was not possible to obtain blood samples in post1. Two samples during tSMS were excluded from the $\mathrm{S} 100$ analyses as outliers. Two additional samples in post 2 were excluded from the NSE analyses because of a visually evident hemolysis (these samples were included in the S100 analyses). Thus, the final serum sample size ( $n$ ) was: baseline $n=17$, during $n=16(n=14$ for $S 100)$, post $1: n=15$, post $2: n=15$ $(n=17$ for S100), $24 \mathrm{~h}: n=13$.

\section{Assessment of cognitive and motor performance}

To detect possible side effects, the following investigations were performed before and after the tSMS testing on the same day. The neuropsychological test battery consisted of the Folstein MiniMental Status Examination (MMSE) [10], and a phonemic Verbal Fluency Test [11] during which the letters F, A and S were presented with subjects required to say as many words beginning with the letter as possible during a $60 \mathrm{~s}$ period. Order of letter presentation was counterbalanced across subjects and conditions - pre- and posttesting. Letter fluency scores were based on the number of correct items generated across the three letter conditions. The Nine-Hole peg test (NHPT) was performed to test fine motor skills (manipulative dexterity) and complex visuomotor coordination with the right hand (this test was not performed with the left hand due to the presence of venous catheter). A two-choice Reaction Time (RT) test measured visuomotor speed. The subject was instructed to press the corresponding key to an arrow direction (pointing left or right) appearing on a screen as quickly and accurately as possible. The test consisted of three blocks, each with 120 trials. RTs and error rates were recorded, with the latter determined as a measure of accuracy to look for a possible trade-off between speed and accuracy.

\section{Statistical analyses}

Blood measures (NSE and S100) were compared to baseline with paired $t$-tests. Note that we purposely did not correct for multiple comparisons in order to conservatively maximize the possibility to find significant changes $(P<0.05)$. One-way ANOVA was employed to corroborate any unexpected significant findings. Cognitive measures were assessed with paired $t$-tests, except for data from the two-choice reaction time task, which were analyzed with a two-way repeated-measures ANOVA (with factors hand and time) to fully exploit the statistical power given by two hands. Values are given as mean \pm standard deviation.

\section{Results}

The 2-h tSMS session was well tolerated by all 17 subjects. None of the subjects needed to interrupt or terminate the session due to side effects. One subject reported mild headache and numbness at the end of the tSMS, but was able to complete the study.

\section{Blood tests}

NSE concentrations were not affected by tSMS (Fig. 2A), neither during the stimulation (paired $t$-test, $P=0.74, n=16$ ), immediately after (post1, $P=0.58, n=15$ ), 1 h after (post2, $P=0.98, n=15$ ), nor $24 \mathrm{~h}$ after $(P=0.92, n=13)$. The average NSE concentration in post2, which represents the most relevant time point from a safety perspective, was $4.79 \pm 2.14 \mathrm{ng} / \mathrm{ml}$ (baseline: $4.75 \pm 3.12 \mathrm{ng} / \mathrm{ml}$ ).

S100 concentrations did not increase with tSMS (Fig. 2B). Interestingly, S100 concentrations were actually significantly decreased both during tSMS $(P=0.0014, n=14)$, immediately after tSMS (post1, $P=0.0166, n=15$ ) and $1 \mathrm{~h}$ after (post2, $P=0.0083, n=17$ ), but not 24 h after $(P=0.33, n=13)$. The average $S 100$ concentration in post 2 was $9.98+7.05 \mathrm{ng} / \mathrm{ml}$ (baseline: $14.11+8.54 \mathrm{ng} / \mathrm{ml}$ ). These decrease in $\mathrm{S} 100$ concentrations remained significant when the data were analyzed with a more conservative one-way ANOVA, either using repeated-measures design with only the 11 subjects for which all time points are available $(F(4,40)=5.7, P=0.0009$ ), or an independent-measures design with all subjects and all available time points $(F(4,71)=3.2, P=0.0178)$.

\section{Cognitive tests}

In the two-choice reaction time task, RTs were faster with the right hand, as expected (two-way ANOVA, hand: $F(1,16)=24.0$, $P=0.0002$ ), but tSMS did not induce any significant effect (time: $F(2,32)=1.5, P=0.24$; interaction: $F(2,32)=1.5, P=0.24$; Fig. $3 \mathrm{~A})$. The number of errors made by the subjects during the task was slightly higher with the right hand (hand: $F(1,16)=4.8, P=0.0440$ ) and again was not affected by tSMS (time: $F(2,32)=1.3, P=0.29$; interaction: $F(2,32)=0.9, P=0.41$; Fig. $3 \mathrm{~B}$ ). 

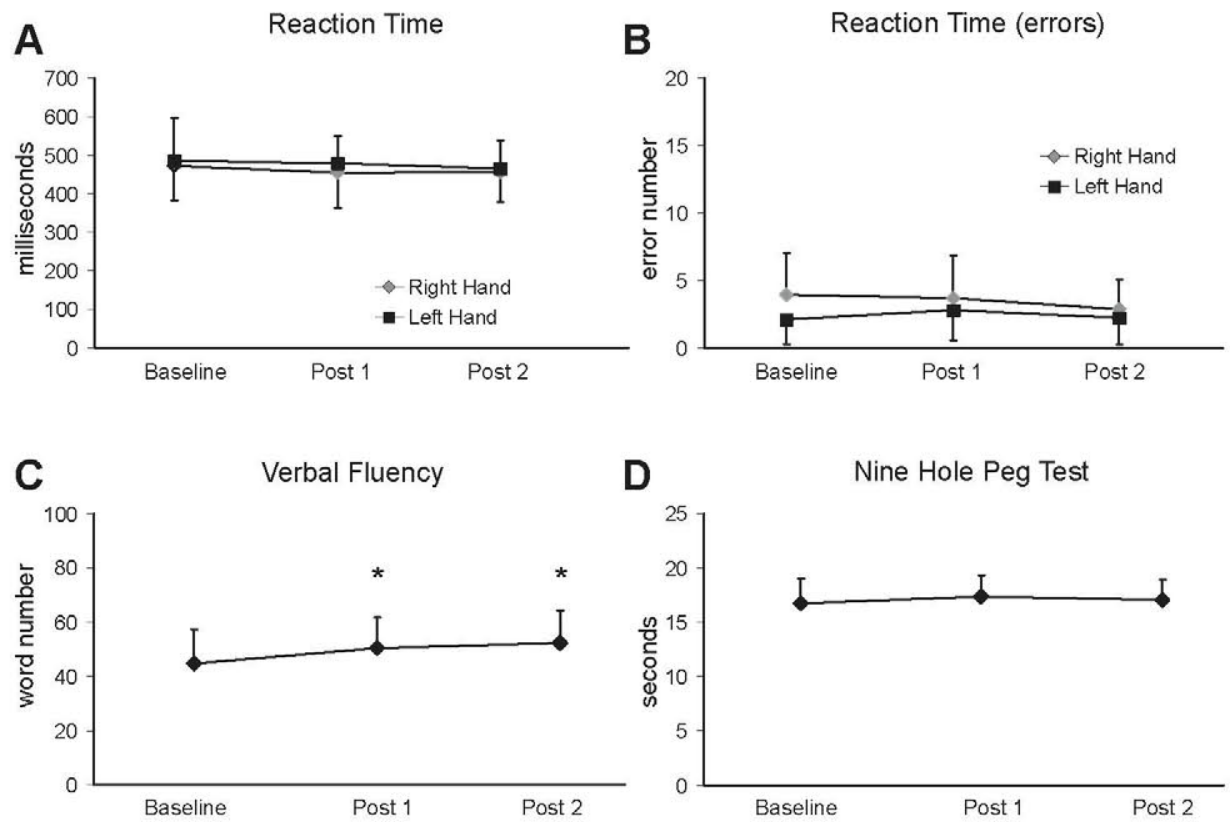

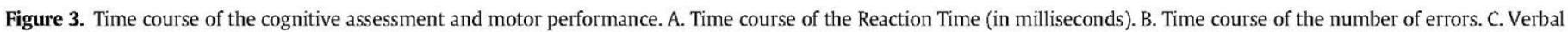
Fluency Test. Time course of the total words counting. D. Time course of the Nine Hole Peg Test. * $=P<0.05$ (paired $t$-test). Error bars indicate standard deviations.

Word fluency was not impaired by tSMS (Fig. 3C). There was actually an improvement at post1 (paired $t$-test, $P=0.0091$ ) and at post2 $(P=0.0026)$, which was confirmed by a more conservative one-way ANOVA $(F(2,32)=7.9, P=0.0016)$, probably reflecting a slight learning effect.

The time to perform the NHPT was not affected by tSMS $(P>0.22$; Fig. 3D). MMSE did not change after tSMS (baseline $=29.3 \pm 1.1$; post $1=29.6 \pm 0.6$; post $2=29.6 \pm 0.8$; $P>0.33$, data not shown in figures).

\section{Discussion}

The principal finding of this study was that $2 \mathrm{~h}$ of tSMS over the occipital cortex appears to be a safe procedure.

There is currently no gold standard for assessing safety for NIBS protocols. At least three types of safety studies can be found in the NIBS literature: (i) studies that evaluate safety a posteriori (similarly to phase 4 clinical trials), assessing the incidence of possible adverse effects in large cohort of patients/subjects that already received specific NIBS protocols (e.g. Refs. [13-15]); (ii) studies that evaluate safety of a known NIBS protocol for its application to a new indication (similarly to phase 1 repositioning clinical trials), assessing the incidence of possible adverse effects in a small cohort of patients (e.g. Refs. [16,17]); (iii) studies assessing safety of a recently-introduced NIBS technique in healthy subjects (e.g. Ref. [18]). Our study belongs to the third group.

The serum level of NSE is a marker of neuronal damage that has been previously used to assess NIBS safety [18-20]. No NSE changes have been reported with cathodal tDCS in 5 normal subjects [19], rTMS in 14 depressed patients [20] and tACS in 8 normal subjects [18]. We also found no changes in NSE levels either during or after tSMS in our experiments. Even though negative results always need to be considered with caution, our study was conservatively designed to achieve higher statistical power compared to previous NIBS safety studies ( $n=15$ for NSE at the most relevant time point, i.e. post2), in order to minimize the risk of type II error.

We also measured serum levels of S100, a marker of glial damage. Only one of the above NIBS safety studies assessed S100 levels, finding no changes [20]. Our a priori hypothesis was that if S100 increased it would have suggested a glial damage and a safety hazard [21]. Surprisingly, we found that the S100 concentration decreased during and after the tSMS. This unexpected result might intriguingly suggest reduced glial reactivity, which could reflect a certain level of tissue protection [21]. However, it needs to be stressed that this is not a definitive finding, and should be confirmed in sham-controlled experiments in order to exclude nonspecific effects [22], which is beyond the scope of the present work. Even though we do not intend to make any strong claims about this unexpected S100 decrease, it does convincingly show that S100 does not increase, strongly supporting the safety of our tSMS protocol.

We observed no significant impairment in performance of visuomotor or cognitive tests. These tests are not typically included in previous NIBS safety studies, but are particularly important in our protocol given the significant behavioral effects recently reported with the application of tSMS to the visual cortex in monkeys [23]. It is important to note that we intended to exclude gross behavioral changes that would be hazardous for the everyday living. Indeed, because tSMS reduces cortical excitability, tSMS is expected to induce subtler behavioral effects associated with the execution of more complex visuomotor/cognitive tasks.

In the present study, we did not perform EEG recordings, which were instead used in previous NIBS safety studies (e.g. Ref. [18]). The rationale for using EEG recordings to assess NIBS safety is because of the increased risk of seizures with protocols that increase cortical excitability. tSMS was shown to reduce cortical excitability both in the sensorimotor cortex of humans [2,3,24], and in the visual cortex of cats and monkeys [23]. Decreased cortical excitability should reduce rather than increase the risk of seizures. In fact, it has been demonstrated that tSMS reduces epileptic activity in animal models $[25,26]$. We therefore argue that EEG recordings are not necessary to establish tSMS safety.

Overall, the purpose of this study was to establish if tSMS could be delivered safely in humans in order to provide safe stimulation patterns for future experimental and therapeutic trials. We confirmed the safety of static magnet fields within the 
recommended limits reported by the WHO (http://www.who.int/ peh-emf/publications/facts/fs299/en/index.html). However, it cannot be excluded that stimulation parameters different from ours (different gradient shape, magnetic field orientation, duration, etc.) might have different safety profiles.

\section{Acknowledgments}

The authors thank A.M. Escobar-Corroto and M. Peñas de la Iglesia for valuable technical support.

\section{References}

[1] Schulz R, Gerloff C, Hummel FC. Non-invasive brain stimulation in neurological diseases. Neuropharmacology 2013;64:579-87.

[2] Oliviero A, Mordillo-Mateos L, Arias P, Panyavin I, Foffani G, Aguilar J. Transcranial static magnetic field stimulation of the human motor cortex. J Physiol 2011;589:4949-58.

[3] Silbert BI, Pevcic DD, Patterson HI, Windnagel KA, Thickbroom GW. Inverse correlation between resting motor threshold and corticomotor excitability after static magnetic stimulation of human motor cortex. Brain Stimul 2013;6:817-20.

[4] Rosen A. Mechanism of action of moderate-intensity static magnetic fields on biological systems. Cell Biochem Biophys 2003;39:163-73.

[5] Coots A, Shi R, Rosen A. Effects of a 0.5-T static magnetic field on conduction in guinea pig spinal cord. J Neurol Sci 2004:222:55-7.

[6] Rosen $\mathrm{AD}$, Lubowsky J. Magnetic field influence on central nervous system function. Exp Neurol 1987;95:679-87.

[7] Rivadulla C, Foffani G, Oliviero A. Magnetic field strength and reproducibility of Neodymium magnets useful for transcranial static magnetic field stimulation of the human cortex. Neuromodulation 2014;17:438-41.

[8] MHRA. Safety guidelines for magnetic resonance imaging equipment in clinical use. 2014 http://www.mhra.gov.uk/Publications/Safetyguidance/ DeviceBulletins/CON2033018.

[9] Persson I, Hardemark HG, Gustafsson J, et al. S-100 protein and neuronspecific enolase in cerebrospinal fluid and serum: markers of cell damage in human nervous system. Stroke 1987;18:911-8.

[10] Folstein M, Folstein SE, McHugh PR. "Mini-Mental State" a practical method for grading the cognitive state of patients for the clinician. J Psychiatr Res $1975 ; 12: 189-98$.
[11] Tranel D, Bigler ED, Howieson DB, Lezak MD. Neuropsychological assessment. Oxford University Press; 2012, ISBN 9780195395525.

[12] Oldfield RC. The assessment and analysis of handedness: the Edinburgh inventory. Neuropsychologia 1971;9:97-113.

[13] Machii K, Cohen D, Ramos-Estebanez C, Pascual-Leone A. Safety of rTMS to non-motor cortical areas in healthy participants and patients. Clin Neurophysiol 2006; 117:455-71.

[14] Poreisz C, Boros K, Antal A, Paulus W. Safety aspects of transcranial direct current stimulation concerning healthy subjects and patients. Brain Res Bull 2007;72:208-14

[15] Brunoni AR, Amadera J, Berbel B, Volz MS, Rizzerio BG, Fregni F. A systematic review on reporting and assessment of adverse effects associated with transcranial direct current stimulation. Int J Neuropsychopharmacol 2011;14:1133-45.

[16] Chae JH, Nahas Z, Wassermann E, Sallee FR, George MS. A pilot safety study of repetitive transcranial magnetic stimulation (rTMS) in Tourette's syndrome. Cogn Behav Neurol 2004;17:109-17.

[17] Carey JR, Evans CD, Anderson DC, Pascual-Leone A. Safety of 6-Hz primed lowfrequency rTMS in stroke. Neurorehabil Neural Repair 2008;22:185-92.

[18] Chaieb L, Antal A, Pisoni A, Focke N, Paulus W. Safety of $5 \mathrm{kHz}$ tACS. Brain Stimul 2014;7:92-6.

[19] Nitsche MA, Nitsche MS, Klein CC, Tergau F, Rothwell JC, Paulus W. Level of action of cathodal DC polarisation induced inhibition of the human motor cortex. Clin Neurophysiol 2003;114:600-4.

[20] Ullrich H, Kranaster L, Sigges E, Andrich J, Sartorius A. Neuron specific enolase and serum remain unaffected by ultra high frequency left prefrontal transcranial magnetic stimulation in patients with depression: a preliminary study. J Neural Transm 2013;120:1733-6.

[21] Donato R, Cannon BR, Sorci G, Geczy CL. Functions of S100 proteins. Curr Mol Med 2013;13:24-57.

[22] Nogueira MI, Abbas SY, Campos IG, Allemandi W, Lawson P, Takada SH, et al. S100beta protein expression: gender- and age-related daily changes. Neurochem Res 2009;34:1355-62.

[23] Aguila J, Cudeiro J, Rivadulla C. Effects of static magnetic fields on the visua cortex: reversible visual deficits and reduction of neuronal activity. Cereb Cortex 2014 [Epub ahead of print].

[24] Kirimoto H, Tamaki H, Matsumoto T, Onishi H. Effect of transcranial static magnetic field stimulation over the sensorimotor cortex on somatosensory evoked potentials in humans. Brain Stimul 2014 Nov-Dec;7(6):836-40.

[25] McLean MJ, Engström S, Holcomb RR, Sanchez D. A static magnetic field modulates severity of audiogenic seizures and anticonvulsant effects of phenytoin in DBA/2 mice. Epilepsy Res 2003;55:105-16.

[26] Mclean MJ, Engström S, Qinkun Z, Spankovich C, Polley DB. Effects of a static magnetic field on audiogenic seizures in black Swiss mice. Epilepsy Res 2008;80:119-31. 